\title{
Herschel/PACS spectroscopy of trace gases of the stratosphere of Titan $\star$
}

\author{
M. Rengel ${ }^{1}$, H. Sagawa ${ }^{1, \star \star}$, P. Hartogh ${ }^{1}$, E. Lellouch ${ }^{2}$, H. Feuchtgruber ${ }^{3}$, R. Moreno ${ }^{2}$, C. Jarchow ${ }^{1}$, R. Courtin ${ }^{2}$, \\ J. Cernicharo ${ }^{4}$, and L. M. Lara ${ }^{5}$ \\ 1 Max-Planck-Institut für Sonnensystemforschung, Max-Planck-Str. 2, 37191 Katlenburg-Lindau, Germany \\ e-mail: rengel@mps.mpg.de \\ 2 LESIA - Observatoire de Paris, CNRS, Université Paris 6, Université Paris-Diderot, 5 place Jules Janssen, 92195 Meudon, France \\ 3 Max-Planck-Institut für extraterrestrische Physik, Giessenbachstrasse, 85748 Garching, Germany \\ ${ }^{4}$ Departamento de Astrofísica, Centro de Astrobiología, CSIC-INTA, Torrejón de Ardoz, 28850 Madrid, Spain \\ 5 Instituto de Astrofísica de Andalucía (CSIC), 18008 Granada, Spain
}

Received 22 May 2013 / Accepted 6 November 2013

\begin{abstract}
Aims. We investigate the composition of Titan's stratosphere from new medium-resolution far-infrared observations performed with the full range of Herschel's Photodetector Array Camera and Spectrometer (PACS) (51-220 $\mu \mathrm{m}$ at a resolution $\lambda / \Delta \lambda$ ranging from 950 to 5500 depending on wavelength and grating order).

Methods. Using PACS, we obtained the spectral emission of several features of the Titan's stratosphere. We used a line-by-line radiative transfer code and the least-squares fitting technique to infer the abundances of the trace constituents.

Results. Numerous spectral features attributable to $\mathrm{CH}_{4}, \mathrm{CO}, \mathrm{HCN}$, and $\mathrm{H}_{2} \mathrm{O}$ are present. From the flux density spectrum measured and by a detailed comparison with synthetic spectra, we constrain the stratospheric abundance of $\mathrm{CH}_{4}$, which is assumed to be constant with altitude, to be $1.29 \pm 0.03 \%$. Similarly, we constrain the abundance of CO to be $50 \pm 2 \mathrm{ppm}$, and the HCN vertical distribution consistent with an increase from $40 \mathrm{ppb}$ at $\sim 100 \mathrm{~km}$ to $\sim 4 \mathrm{ppm}$ at $\sim 200 \mathrm{~km}$, which is an altitude region where the HCN signatures are sensitive. Measurements of three $\mathrm{H}_{2} \mathrm{O}$ rotational lines confirm the $\mathrm{H}_{2} \mathrm{O}$ distribution profile recently obtained with Herschel. Furthermore, we determine the isotopic ratios ${ }^{12} \mathrm{C} /{ }^{13} \mathrm{C}$ in $\mathrm{CO}$ and $\mathrm{HCN}$ to be $124 \pm 58$, and $66 \pm 35$, respectively. Comparisons between our results and the values derived with other instruments show that our results are consistent with the vertical distributions and isotopic ratios in previous studies, except for the HCN distribution obtained with Cassini/CIRS, which does not agree with the PACS lines at the $1-\sigma$ confidence interval.
\end{abstract}

Key words. planets and satellites: atmospheres - planets and satellites: individual: Titan - techniques: spectroscopic

\section{Introduction}

The nitrogen $\left(N_{2}\right)$-dominated atmosphere of Titan, Saturn's largest moon, exhibits a great diversity and complexity of molecules and high organic material abundances. Many species are formed in the upper atmosphere (above $600 \mathrm{~km}$ ) by interaction of solar UV photons, energetic ions/electrons, and cosmicray particles, which initiate a complicated photochemical network. For several of these molecules, the region between the upper atmosphere and their condensation sink (60-100 km) is a transition region. In the stratosphere $(40-320 \mathrm{~km})$, complex chemistry is still in action, and this region is probed mainly with thermal infrared measurements. Previous studies of Titan's submillimetre and infrared spectra from space (e.g. the Infrared Space Observatory (ISO) and Cassini/Composite InfraRed Spectrometer (CIRS)) and ground-based facilities at different spectral resolutions have revealed many specific aspects of the molecular composition, permitted a detailed investigation of the photochemical and dynamical processes in Titan's

\footnotetext{
* Herschel is an ESA space observatory with science instruments provided by European-led Principal Investigator consortia and with important participation from NASA.

$\star \star$ Current address: National Institute of Information and Communications Technology, Japan.
}

atmosphere, and confirmed the seasonal behaviour of Titan's atmospheric composition (e.g. Roe et al. 2004; de Kok et al. 2007; Coustenis et al. 2007; Teanby et al. 2009a, 2012; Rengel et al. 2011). New observations at different spectral ranges, higher spectral resolution and sensitivity, and at different temporal coverage during a Titan's year (29.5 Earth years) offer the opportunity of exploring and helping to advance the study of the abundance distribution (e.g. new abundance constraints and rate of seasonal variations).

The advent of the Herschel Space Observatory (Pilbratt et al. 2010) allowed to determine the stratosphere abundances of $\mathrm{CH}_{4}, \mathrm{CO}$ and $\mathrm{HCN}$ with the Spectral and Photometric Imaging Receiver (SPIRE) covering the 194-671 $\mu \mathrm{m}$ spectral region with an spectral resolution of $0.04 \mathrm{~cm}^{-1}$ (Courtin et al. 2011), to detect HNC with the Heterodyne Instrument for the Far-Infrared (HIFI; Moreno et al. 2011), and infer the vertical profile of $\mathrm{H}_{2} \mathrm{O}$ over the $100-450 \mathrm{~km}$ altitude range by combining data from HIFI and the Photodetector Array Camera and Spectrometer (PACS; Moreno et al. 2012).

In this paper, we report the results from a full grating resolution spectra of Titan at 51-220 $\mu \mathrm{m}(5878-1363 \mathrm{GHz})$ taken with PACS (Poglitsch et al. 2010) onboard Herschel. Although it covers a smaller spectral range than Cassini/CIRS and a range similar to that of ISO/LSW ([17-1000] and [2.4-196] $\mu \mathrm{m}$, 
Table 1. Summary of the observations.

\begin{tabular}{lccc}
\hline \hline Obs. ID & $\begin{array}{c}\text { Start time } \\
{[\mathrm{UTC}]}\end{array}$ & $\begin{array}{c}T_{\text {obs }} \\
{[\mathrm{s}]}\end{array}$ & $\begin{array}{c}\text { Range }^{a} \\
{[\mu \mathrm{m}]}\end{array}$ \\
\hline 1342199145 & 23 June 2010 00:42:40 & 1140 & $51-073^{B 2 A}, 102-146^{R 1}$ \\
1342199146 & 23 June 2010 01:03:48 & 2268 & $70-105^{B 2 B}, 140-220^{R 1}$ \\
1342211198 & 15 Dec. 2010 05:56:53 & 4051 & $55-072^{B 3 A}, 165-216^{R 1}$ \\
1342211199 & 15 Dec. 2010 07:06:33 & 3509 & $70-099^{B 2 B}, 140-198^{R 1}$ \\
1342211200 & 15 Dec. 2010 08:07:14 & 1983 & $51-071^{B 2 A}, 102-141^{R 1}$ \\
\hline
\end{tabular}

Notes. ${ }^{(a)}$ Spectral band.

respectively), PACS has a higher spectral resolution than previous instruments (ISO/SWS and LSW have resolving powers of 1000-2500 and 200, respectively, and Cassini/CIRS has a spectral resolution of $0.5 \mathrm{~cm}^{-1}$ ). We report the gases identified in the spectra, derive their abundances, and compare them with previous studies. A few isotopic lines are identified, from which we estimate the isotopic ratios ${ }^{12} \mathrm{C} /{ }^{13} \mathrm{C}$ in $\mathrm{CO}$ and $\mathrm{HCN}$. More possible detections of isotopes are limited by the sensitivity of the PACS observations and the weakness of the lines, which can easily be confused with noise.

\section{Observations and data reduction}

In the framework of the program "Water and Related Chemistry in the Solar System", (Hartogh et al. 2009), fullrange observations of Titan were performed on June 23 and December 15, 2010 (Table 1). Observations were carried out in chopped-nodded PACS range spectroscopy modes (Poglitsch et al. 2010), at Nyquist/spectral energy distribution (SED; second and first orders of the grating spectrometer) and at high spectral sampling density (third and first orders) for the June and December observations, respectively.

The entire spectral range of PACS was measured at full instrumental resolution $\lambda / \Delta \lambda$, ranging from 950 to 5500 depending on wavelength and grating order (Poglitsch et al. 2010). Since blue and red spectrometer data are acquired in parallel, several spectral ranges were observed in overlap. To minimise spectral contributions from Saturn, Titan was observed at the largest feasible elongations from Saturn (168" and 173" on 23 June and 15 December 2010, respectively). The data processing from Level 0 to 1 was carried out by standard PACS pipeline modules using the Herschel interactive processing environment (HIPE v.4 and v.6). All subsequent processing (flat-fielding, outlier removal and rebinning) was carried out with standard interactive data language (IDL) tools. Because the absolute calibration accuracy is limited by detector response drifts and slight pointing offsets ${ }^{1}$, the resulting spectrum was divided by its continuum, a step that is not subject to changes through later HIPE releases. For spectral ranges covered more than once, the observation with the highest resolution was selected. When observations are covered with the same spectral band, an averaged observation was used to increase the signal-to-noise-ratio $(\mathrm{S} / \mathrm{N})$. The data adquired during different operational days were carefully compared to verify that no remarkable temporal changes in the instrumental characterists and in seasonal changes in the atmosphere of Titan ocurred. Within the noise level, no variations were seen.

\footnotetext{
1 http://herschel.esac.esa.int/
}

The composite spectrum is shown in Fig. 1. It shows several emission molecular signatures attributable to $\mathrm{CH}_{4}, \mathrm{CO}, \mathrm{HCN}$, and $\mathrm{H}_{2} \mathrm{O}$, molecules that have been previously detected on Titan, but most line transitions are detected for the first time. We detected some faint emission features attributable to isotopologues above the noise (3-5\% above the line-to-continuum ratio), the ${ }^{13} \mathrm{CO}(15-14)$ and (16-15), and the $\mathrm{H}^{13} \mathrm{CN}(19-18)$ and $(20-19)$ spectral lines.

\section{Modelling the Titan PACS spectra and comparing them with the observed spectra}

Our simulation package was developed based on the general forward and inversion model called microwave observation line estimation and retrieval (MOLIERE (v5); Urban et al. 2004; Rengel et al. 2010; Sagawa et al. 2010). The forward model consists of a line-by-line radiative transfer model that takes into consideration a homogeneous spherically symmetric atmosphere of Titan (altitudes from 0 to $800 \mathrm{~km}$ ) and instrumental functions (where the Herschel beam profile and the PACS spectral response are considered, we assumed a uniform brightness temperature on the disk and Titan as a point-source). $\mathrm{CH}_{4}, \mathrm{CO}, \mathrm{HCN}$, and $\mathrm{H}_{2} \mathrm{O}$ (and their isotopologues) were considered for the line opacity calculation. Isotopic ratios were initially set by adopting the values derived by Courtin et al. (2011), when available, and terrestrial ratio ones. For the thermal profile of Titan's atmosphere, we adopted the distribution used in Moreno et al. (2011), a combination of the Huygens Atmospheric Structure Instrument (HASI) profile (Fulchignoni et al. 2005) below $140 \mathrm{~km}$, and Cassini/CIRS stratospheric temperatures (Vinatier et al. 2010) above $140 \mathrm{~km}$.

The a priori molecular mixing ratios adopted in this study are those derived by Niemann et al. (2010) and de Kok et al. (2007) for $\mathrm{CH}_{4}$ and $\mathrm{CO}$, respectively, and the a priori profile is that derived by Marten et al. (2002) for HCN. Spectroscopic parameters such as the transition frequency and the line intensity were derived from the HITRAN 2008 compilation (Rothman et al. 2009), and line strengths for $\mathrm{CH}_{4}$ were taken from Boudon et al. (2010). As previously mentioned, we analysed the PACS spectra on the basis of the line-to-continuum ratios. We used the collision-induced absorption (CIA) coefficients of $\mathrm{N}_{2}$ and $\mathrm{CH}_{4}$ based on the formulation of Borysow \& Frommhold (1986), Borysow \& Frommhold (1987), and Borysow \& Tang (1993). A comparison between our forward model and that reported in Moreno et al. (2012) under the condition of a pencil beam (a one-dimensional beam, i.e., the beam width is zero) in the nadir viewing geometry shows that they agree well within $3 \%$. The PACS spectral response modelling $(\lambda / \Delta \lambda \sim 1000-5000$, which depends on the wavelength and grating diffraction conditions) was performed by following the descriptions by Poglitsch et al. (2010), and the related documents on the Herschel Science Center ${ }^{1}$. A comparison between observed data and synthetic spectra is shown in Fig. 1.

The best-fit between the observed and modelled spectra was performed simultaneously by least-squares fitting, that is, minimising $\chi^{2}$. As sources of uncertainties we considered measurement and modelling errors. While the first one was considered as the standard deviation of several ObsIds (when available) and the oscillations about the smooth spectral continuum, modelling errors were considered as the variance caused by the residual between the measured and fitted fluxes, set it with a value of $3 \%$ and $1 \%$ for the blue and red bands, respectively. We 

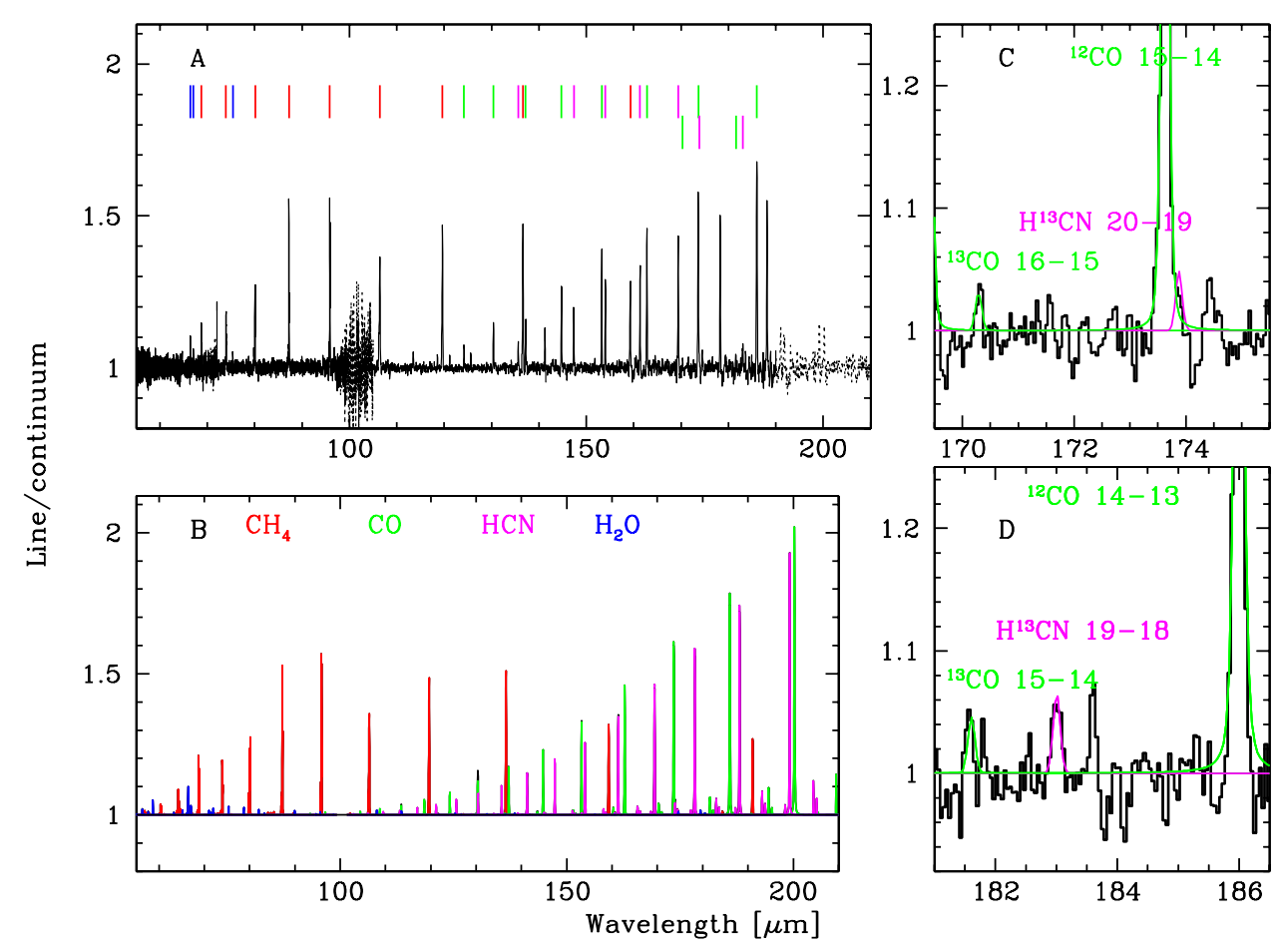

Fig. 1. A) black line shows the PACS spectrum of Titan, expressed in line-to-continuum ratios. The coloured marks at the top show the selected lines and transitions for the analysis in Sect. 4. The dashed line indicates spectral regions affected by spectral leakage due to grating order overlap. B) best-fit synthetic spectra indicating the signatures attributable to $\mathrm{CH}_{4}, \mathrm{CO}, \mathrm{H}_{2} \mathrm{O}$, and $\mathrm{HCN}$ (Sect. 4). C)-D) the [170-176] and [181-187] $\mu \mathrm{m}$ regions of the PACS spectrum displaying signatures of the isotopologues, along with the best-fit model with the detected isotopic variants.

quote our results at $1-\sigma$ significance level, which is given by a change $\Delta \chi^{2}=\chi^{2}-\chi_{\min }^{2}$ of 1 .

\section{Mixing ratios of individual trace constituents}

$\mathrm{CH}_{4}: \mathrm{CH}_{4}$ is the second-most abundant gas in Titan's atmosphere, and its origin is unknown. Possible scenarios range from a continuous resupply to the atmosphere from a reservoir (from Titan's interior) to a recent and transitory component of the atmosphere (Nixon et al. 2012). The $\mathrm{CH}_{4}$ distribution has been determined previously, it is uniformly mixed in the entire stratosphere (Niemann et al. 2005), and abundances of $1.6 \pm 0.5 \%, 1.48 \pm 0.09 \%$ between 76 and $140 \mathrm{~km}$, and $1.33 \pm 0.07 \%$ were measured previously (Flasar et al. 2005; Niemann et al. 2010; Courtin et al. 2011), respectively. We used selected $\mathrm{CH}_{4}$ lines, simulated the observed spectra using the forward model (adopting several constant abundance distributions), and determined the best-fit (Figs. 2, 3). We found a best-fit volume-mixing ratio of $1.29 \pm 0.03 \%$, which is consistent with the SPIRE determination.

CO: $\mathrm{CO}$ is the fourth-most abundant molecule in Titan's atmosphere. It is not established whether the $\mathrm{CO}$ in Titan's atmosphere is primordial (from the interior or surface, Wilson \& Atreya 2004) or due solely to external sources. As first shown by Hörst et al. (2008), the influx of $\mathrm{O}^{+}$from Saturn's magnetosphere into Titan (Hartle et al. 2006) can lead to the formation of $\mathrm{CO}$ through reactions between ground-state $\mathrm{O}\left({ }^{3} \mathrm{P}\right)$ and $\mathrm{CH}_{3}$. Recent observations suggest that $\mathrm{CO}$ in Titan is uniformly mixed throughout Titan's atmosphere (Gurwell 2004; Baines et al. 2006; de Kok et al. 2007). Such a profile can be expected if its long chemical lifetime in the oxygen-poor Titan atmosphere is considered $\left(\sim 10^{9}\right.$ years, Yung et al. 1984). In this study, we retrieved the $\mathrm{CO}$ abundance by assuming a vertically

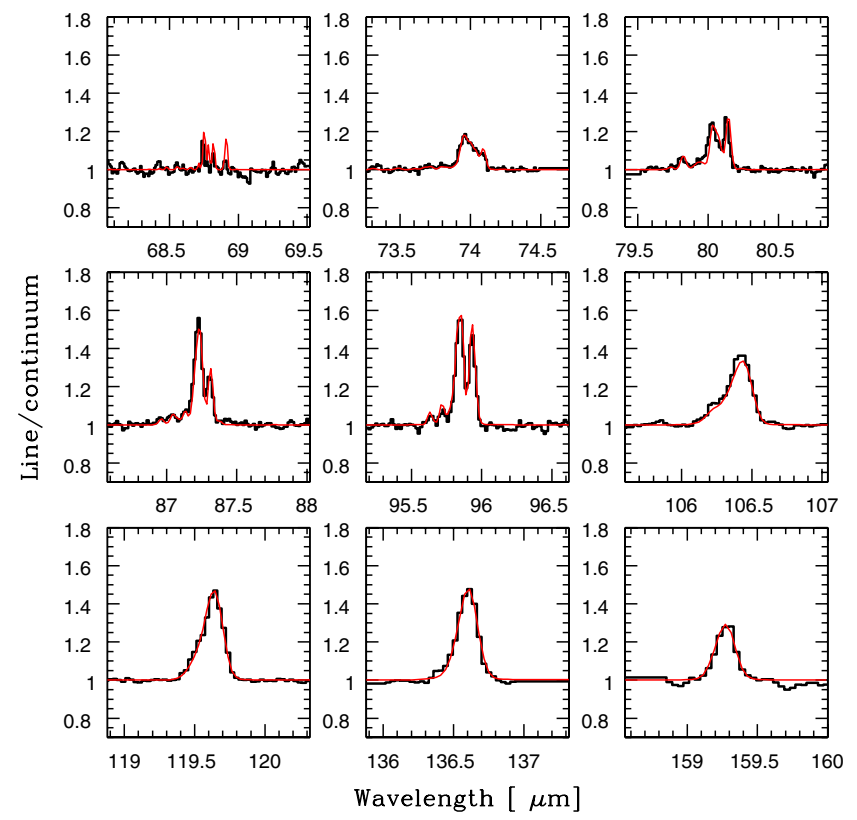

Fig. 2. Observed and best-fit simulated $\mathrm{CH}_{4}$ lines (black and red, respectively).

constant profile and simultaneously computed synthetic selected CO lines for several abundances. We found a best-fit volume-mixing ratio of $50 \pm 2 \mathrm{ppm}$ (Fig. 4), consistent with the results from Herschel/SPIRE of $40 \pm 5$ ppm (Courtin et al. 2011), Cassini/CIRS (de Kok et al. 2007) of $47 \pm 8$ ppm, the Atacama Pathfinder EXperiment (APEX)/ Swedish Heterodyne Facility Instrument (SHeFI) of $30_{-8}^{+15} \mathrm{ppm}$ (Rengel et al. 2011), and the Submillimeter Array (SMA) of $51 \pm 4$ ppm (Gurwell et al. 2012). The PACS CO lines reported here are sensitive to 


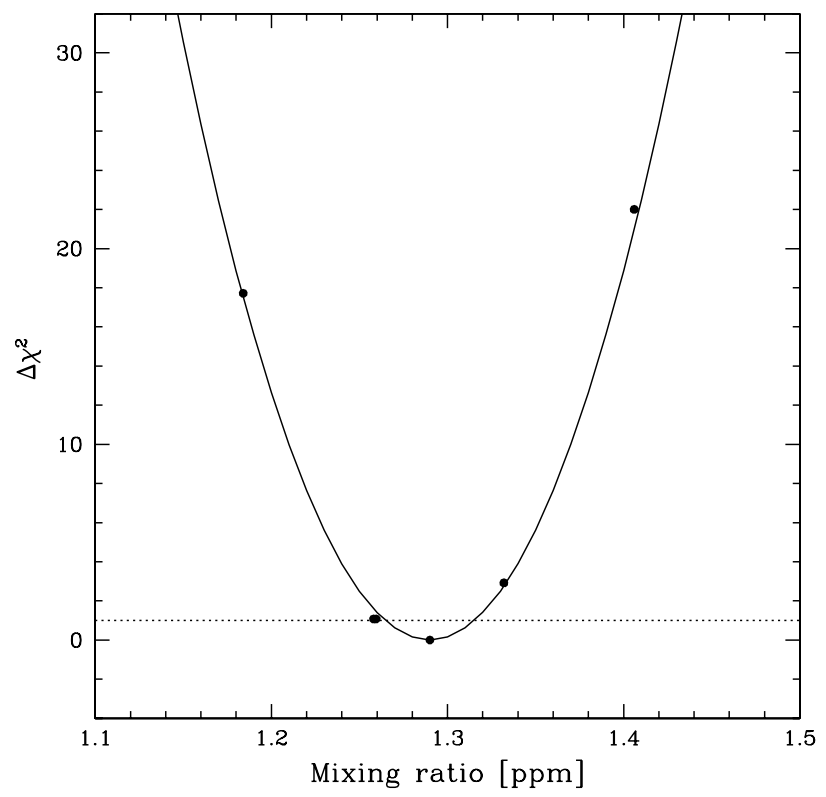

Fig. 3. Best-fit volume-mixing ratio and the $1-\sigma$ confidence limits.
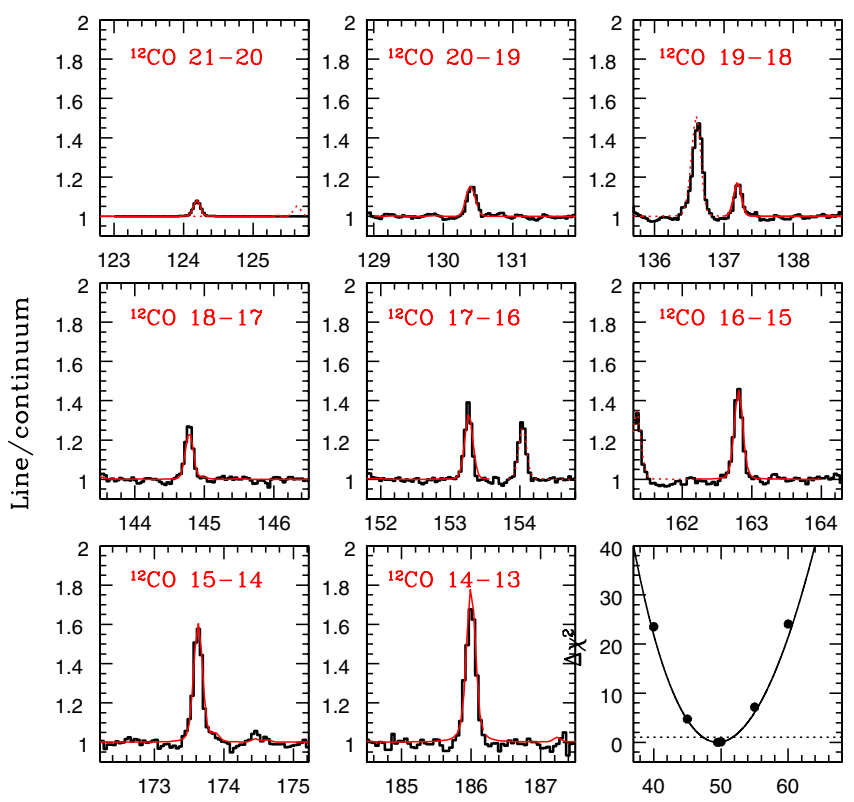

Wavelength $[\mu \mathrm{m}]$ Mixing ratio $[\mathrm{ppm}]$

Fig. 4. Observed and best-fit simulated CO lines (black and red, respectively). The dashed lines show the best-fitting simulated lines of other species. Note that the observed CO line at $130.37 \mu \mathrm{m}$ is overlapped by the $\mathrm{HCN}$ line at $130.24 \mu \mathrm{m}$. Bottom-right panel: best-fit volume-mixing ratio and the $1-\sigma$ confidence limits.

the $[60-170] \mathrm{km}$ range altitude.

HCN: HCN is generated photochemically in Titan's atmosphere from reactions of hydrocarbon radicals with atomic nitrogen. The latter is produced from EUV or electron impact on $\mathrm{N}_{2}$, or possibly liberated as a result of cometary impacts (Sekine et al. 2011). HCN is produced above $300 \mathrm{~km}$ (Lara et al. 1996; Wilson \& Atreya 2004) and removed by condensation deeper in the atmosphere. This sets up a concentration gradient. A more recent alternative suggests that $\mathrm{HCN}$ is thermodynamically generated via a shock chemistry under lightning discharges in the low atmosphere (Kovács \& Turányi 2010). We analysed the
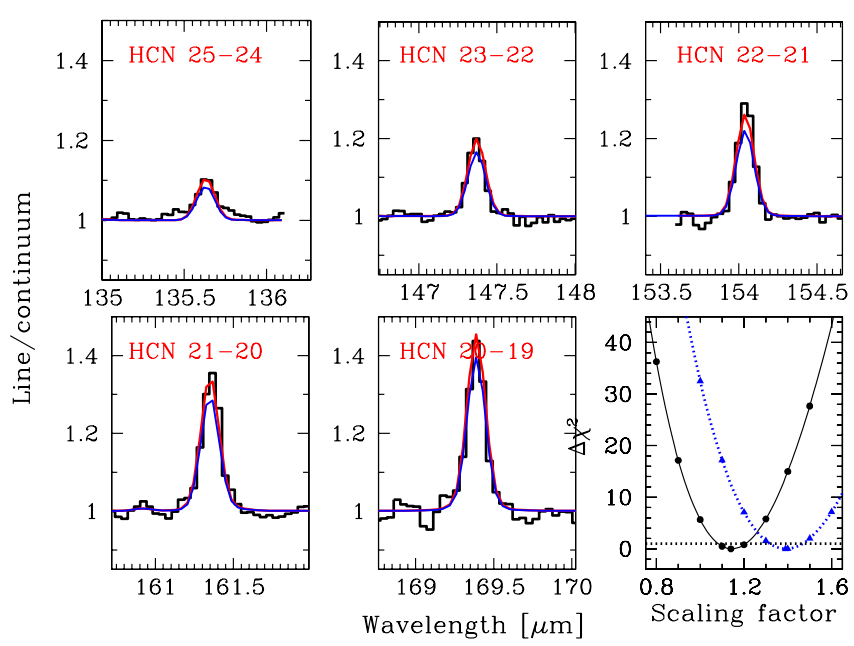

Fig. 5. Observed and best-fit simulated HCN lines (black and red, respectively). Blue spectra indicate the simulated spectra using the abundance profile derived from Vinatier et al. (2007).

observed spectra by scaling a well-probed reference distribution with a vertically constant factor. This offers a reliable result under the current data quality and has also been successfully applied for the Herschel/SPIRE observations (Courtin et al. 2011), which also facilitates the inter-comparisons. We retrieved the scaling factor applied to the adopted distribution of Marten et al. (2002), minimising $\chi^{2}$, and using a simultaneous combination of five $\mathrm{HCN}$ lines (selected as good-quality lines in terms of the baseline corrections). We obtained a scaling factor of $1.14 \pm 0.06$ as a best-fit to the observed spectrum (Fig. 5), consistent with the value of $1.02 \pm 0.13$ derived with SPIRE (Courtin et al. 2011). For comparisons the simulated lines following the distribution obtained by Cassini/CIRS (Vinatier et al. 2007) are also shown. The CIRS distribution disagrees with the PACS observations at the 1- $\sigma$ level. We discarded the difference in geometry as the source of the inconsistency because a disk-averaged spectrum gives preferential weight to low latitudes. Although CIRS data at southern latitudes show no variation during 3.5 years (Teanby et al. 2009b), temporal variability as the source of discrepancy is not completely ruled out: some scatter in the HCN values in Teanby et al. (2009b) is compatible with the differences we notice, and the PACS and CIRS data were not recorded in the same period. The absolute abundance in the CIRS data seems to be a possible source because by multiplying the Vinatier et al. (2007) distribution by factor of 1.39 a good fit is recovered. The analysis presented here confirms the results from Marten et al. (2002). Figure 6 shows our retrieved $\mathrm{HCN}$ vertical distribution (sensitive to an altitude between 100 and $200 \mathrm{~km}$ ) compared with the distributions obtained with Cassini/CIRS and Herschel/SPIRE.

$\mathrm{H}_{2} \mathrm{O}$ : the origin of Titan's water remains puzzling. Based on HIFI observations of the $\mathrm{H}_{2} \mathrm{O}$ Enceladus torus, Hartogh et al. (2011) found that Enceladus is a source for Titan water, but not sufficient given the early estimates of the influx $\mathrm{OH} / \mathrm{H}_{2} \mathrm{O}$ fluxes into Titan as summarized by Strobel (2010). By using combined PACS and HIFI observations of water lines in Titan, covering several periods in 2010 and 2011 and with a higher $\mathrm{S} / \mathrm{N}$, Moreno et al. (2012) determined the $\mathrm{H}_{2} \mathrm{O}$ profile, and their estimates of the $\mathrm{OH} / \mathrm{H}_{2} \mathrm{O}$ now offer a possible reconciliation with the input flux into Titan caused by the plume activity of 
M. Rengel et al.: Herschel/PACS spectroscopy of trace gases of the stratosphere of Titan

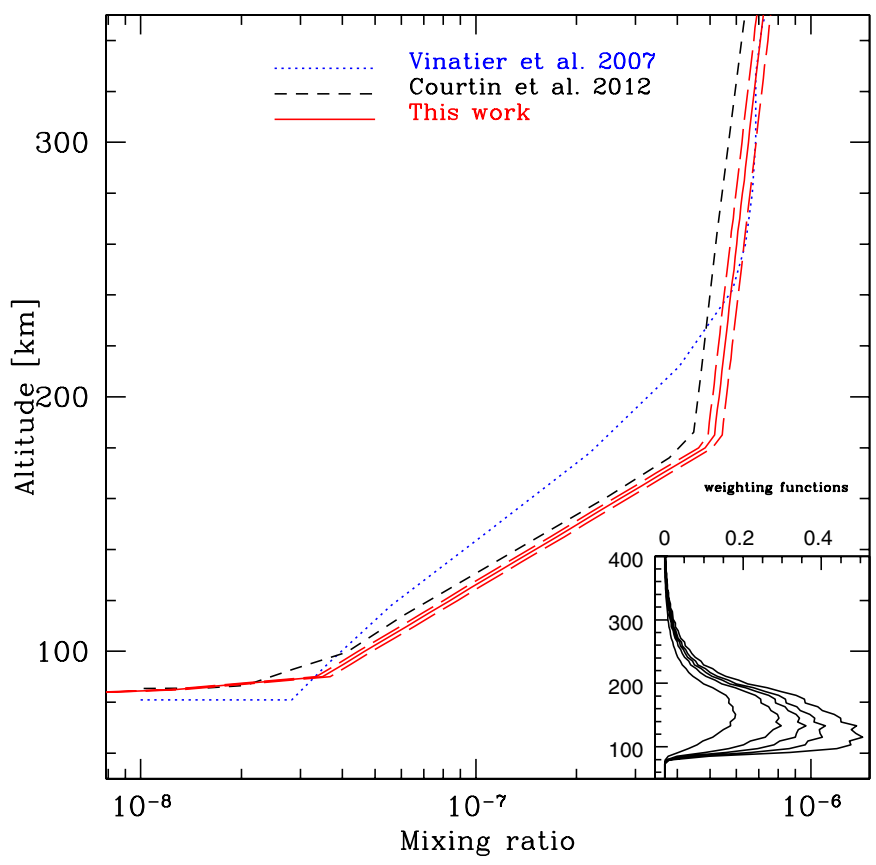

Fig. 6. Distribution of HCN (solid line) compared with the profile obtained by Vinatier et al. (2007) from Cassini/CIRS data at $15^{\circ} \mathrm{S}$ latitude (dotted line) and by Courtin et al. (2011) from Herschel/SPIRE data (short dashes). Long-dashed lines show the 1- $\sigma$ limit. The curves in the small box represent the weighting functions for each line. We used the same line order as in Fig. 5 (from left to right).

Enceladus. Measurements of water vapor in Titan's stratosphere have also been recently reported using Cassini/CIRS (Cottini et al. 2012). Both Cassini and Herschel observations indicate that the previous models over-predict the water abundance in Titan's lower stratosphere, but the two vertical abundance differ. The photochemical model developed by Krasnopolsky (2012) yields a $\mathrm{H}_{2} \mathrm{O}$ profile that includes the $\mathrm{O}^{+}$flux. This profile lies between the Herschel (Moreno et al. 2012) and Cassini (Cottini et al. 2012) observations. We measured the transitions $\mathrm{H}_{2} \mathrm{O}\left(3_{30}-2_{21}\right), \mathrm{H}_{2} \mathrm{O}\left(3_{31}-2_{20}\right)$, and $\mathrm{H}_{2} \mathrm{O}\left(3_{21}-2_{12}\right)$ at $66.6,67.1$ and $75.4 \mu \mathrm{m}$, respectively. The $\mathrm{H}_{2} \mathrm{O}\left(3_{31}-2_{20}\right)$ one is measured for first time. We simulated the spectra considering the semiempirical $\mathrm{S}_{a}$ profile determined in Moreno et al. (2012). Figure 7 shows a comparison between the observed and modelled $\mathrm{H}_{2} \mathrm{O}$ lines. $\mathrm{S}_{a}$ fits the line at $66.4 \mu \mathrm{m}$, but underestimates the lines at 67.1 and $75.4 \mu \mathrm{m}$ by a factor of $\sim 0.67$. Considering the $\mathrm{S} / \mathrm{N}$ limitations, we focused on the $66.4 \mu \mathrm{m}$ line, and the $\mathrm{S}_{a}$ distribution is compatible with these PACS lines. Additional sources of discrepancies are the intensity variations within $10 \%$, the differences of the radiative description among the forward models, and errors due to the incomplete radiative description.

Isotopic ratios: ${ }^{13} \mathrm{C} /{ }^{12} \mathrm{C}$ in $\mathrm{CO}$ and $\mathrm{HCN}$ : the detection of the isotopes ${ }^{13} \mathrm{CO}(15-14)$ and $(16-15)$, and $\mathrm{H}^{13} \mathrm{CN}(19-18)$ and (20-19) is only marginal (Fig. 1). Their abundances are found by a least-squares fit, with errors at the 1- $\sigma$ significance level. The derived isotopic ratio estimates are ${ }^{12} \mathrm{C} /{ }^{13} \mathrm{C}$ of $124 \pm 58$, and $66 \pm 35$ in $\mathrm{CO}$ and $\mathrm{HCN}$, respectively. They are consistent with the terrestrial value of 89.3 given the error bars. The ${ }^{12} \mathrm{C} /{ }^{13} \mathrm{C}$ from this work is consistent with the measurements by SPIRE, CIRS, and SMA (Courtin et al. 2011; Vinatier et al. 2007; Gurwell 2004, 2008).
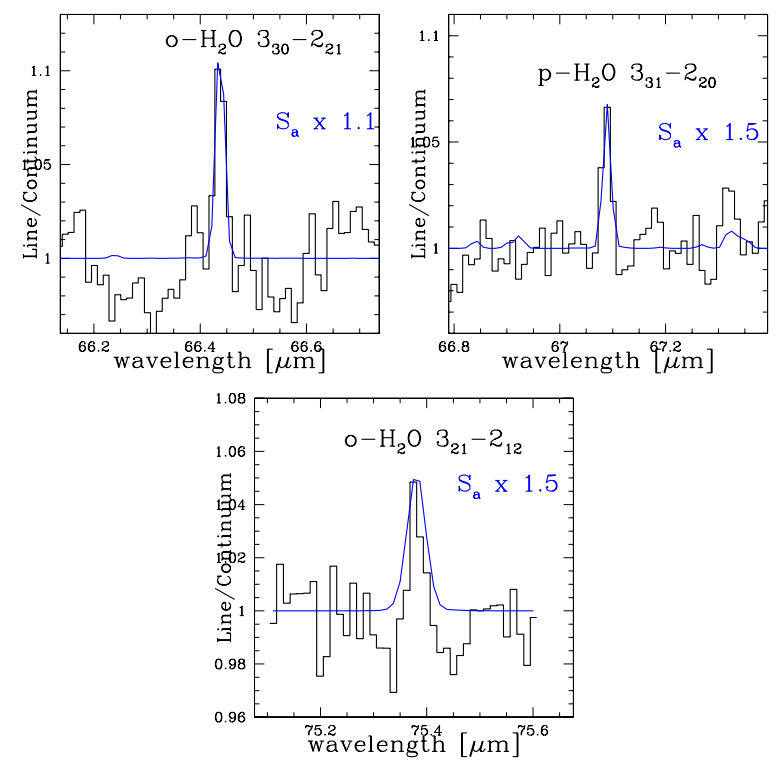

Fig. 7. Comparisons between synthetic spectra computed with the semiempirical $\left(S_{a}\right)$ profile of water (Moreno et al. 2012) and an ensemble of three PACS lines at 66.44, 67.09 (ObsId 1342211198) and $75.38 \mu \mathrm{m}$ (ObsId 1342211199).

\section{Conclusions}

We reported the first observations obtained with the full range of Herschel/PACS of Titan. PACS produces a new survey of Titan's spectrum between 51 and $220 \mu \mathrm{m}$. Signatures attributable to $\mathrm{CH}_{4}, \mathrm{CO}, \mathrm{HCN}$, and $\mathrm{H}_{2} \mathrm{O}$ are detected.

We investigated the $\mathrm{CH}_{4}, \mathrm{CO}$, and $\mathrm{HCN}$ composition of Titan's stratosphere as seen by Herschel/PACS. Nine lines of $\mathrm{CH}_{4}$ dominate the PACS spectrum and are consistent with an abundance of $1.29 \pm 0.03 \%$, which is consistent with the value obtained by SPIRE and that obtained in situ by the Huygens/Gas Chromatograph Mass Spectrometer (GCMS). Eight rotational lines of CO that dominate the PACS spectrum were selected to determine its abundance. Assuming it is well mixed, its abundance was found to be $50 \pm 2 \mathrm{ppm}$, consistent with previous studies. Five lines of $\mathrm{HCN}$ were selected to retrieve the $\mathrm{HCN}$ vertical distribution. Our HCN mixing-ratio estimations, with a 1.14 scaling factor of the Marten et al. (2002) distribution between 100 and $200 \mathrm{~km}$, are consistent with those with Herschel/SPIRE. The HCN distribution obtained by CIRS does not seem to reproduce the HCN lines observed with PACS well. Within the known sources of errors, the $\mathrm{H}_{2} \mathrm{O}$ distribution proposed from previous Herschel observations produces a satisfactory fit to three $\mathrm{H}_{2} \mathrm{O}$ lines detected. The ${ }^{13} \mathrm{C}$ isotope was detected in $\mathrm{CO}$ and $\mathrm{HCN}$. We determined ${ }^{12} \mathrm{C} /{ }^{13} \mathrm{C}$ in $\mathrm{CO}$ and $\mathrm{HCN}$ on Titan (Sect. 4).

Limb observations of Titan's submillimetre radiation (e.g. in the $300-360,540-660$, and $1080-1280 \mathrm{GHz}$ spectral ranges) are required to detect more rotational lines, measure rotational lines of isotopes, and winds in regions higher than the $200-1200 \mathrm{~km}$ altitude (Lellouch et al. 2010).

Acknowledgements. We thank the mission planners for the scheduling of these observations, L. Rezac for helpful discussions and the anonymous referee for helping to improve the manuscript. PACS has been developed by a consortium of institutes led by MPE (Germany) and including UVIE (Austria); KUL, CSL, IMEC (Belgium); CEA, OAMP (France); MPIA (Germany); IFSI, OAP/AOT, OAA/CAISMI, LENS, SISSA (Italy); IAC (Spain). This development has been supported by the funding agencies BMVIT (Austria), ESA-PRODEX (Belgium), CEA/CNES (France), DLR (Germany), ASI (Italy), and CICT/MCT (Spain). 


\section{References}

Baines, K. H., Drossart, P., Lopez-Valverde, M. A., et al. 2006, Planet. Space Sci., 54, 1552

Borysow, A., \& Frommhold, L. 1986, ApJ, 311, 1043

Borysow, A., \& Frommhold, L. 1987, ApJ, 318, 940

Borysow, A., \& Tang, C. 1993, Icarus, 105, 175

Boudon, V., Pirali, O., Roy, P., et al. 2010, J. Quant. Spec. Radiat. Transf., 111, 1117

Cottini, V., Nixon, C. A., Jennings, D. E., et al. 2012, Icarus, 220, 855

Courtin, R., Swinyard, B. M., Moreno, R., et al. 2011, A\&A, 536, L2

Coustenis, A., Achterberg, R. K., Conrath, B. J., et al. 2007, Icarus, 189, 35

de Kok, R., Irwin, P. G. J., Teanby, N. A., et al. 2007, Icarus, 191, 223

Flasar, F. M., Achterberg, R. K., Conrath, B. J., et al. 2005, Science, 308, 975

Fulchignoni, M., Ferri, F., Angrilli, F., et al. 2005, Nature, 438, 785

Gurwell, M. A. 2004, ApJ, 616, L7

Gurwell, M. A. 2008, in AAS/Division for Planetary Sciences Meeting Abstracts, BAAS, 40, 423

Gurwell, M. A., Butler, B. J., \& Moullet, A. 2012, in AAS/Division for Planetary Sciences Meeting Abstracts, \#312.12, 44

Hartle, R. E., Sittler, E. C., Neubauer, F. M., et al. 2006, Planet. Space Sci., 54, 1211

Hartogh, P., Lellouch, E., Crovisier, J., et al. 2009, Planet. Space Sci., 57, 1596

Hartogh, P., Lellouch, E., Moreno, R., et al. 2011, A\&A, 532, L2

Hörst, S. M., Vuitton, V., \& Yelle, R. V. 2008, J. Geophys. Res.: Planets, 113

Kovács, T., \& Turányi, T. 2010, Icarus, 207, 938

Krasnopolsky, V. A. 2012, Planet. Space Sci., 73, 318

Lara, L. M., Lellouch, E., López-Moreno, J. J., \& Rodrigo, R. 1996, J. Geophys. Res., 101, 23261
Lellouch, E., Vinatier, S., Moreno, R., et al. 2010, Planet. Space Sci., 58, 1724 Marten, A., Hidayat, T., Biraud, Y., \& Moreno, R. 2002, Icarus, 158, 532

Moreno, R., Lellouch, E., Lara, L. M., et al. 2011, A\&A, 536, L12

Moreno, R., Lellouch, E., Lara, L. M., et al. 2012, Icarus, 221, 753

Niemann, H. B., Atreya, S. K., Bauer, S. J., et al. 2005, Nature, 438, 779

Niemann, H. B., Atreya, S. K., Demick, J. E., et al. 2010, J. Geophys. Res. Planets, 115,12006

Nixon, C. A., Temelso, B., Vinatier, S., et al. 2012, ApJ, 749, 159

Pilbratt, G. L., Riedinger, J. R., Passvogel, T., et al. 2010, A\&A, 518, L1

Poglitsch, A., Waelkens, C., Geis, N., et al. 2010, A\&A, 518, L2

Rengel, M., Sagawa, H., \& Hartogh, P. 2010, Adv. Geosci., 19, 335

Rengel, M., Sagawa, H., \& Hartogh, P. 2011, Adv. Geosci., 25, 173

Roe, H. G., de Pater, I., \& McKay, C. P. 2004, Icarus, 169, 440

Rothman, L., Gordon, I., Barbe, A., et al. 2009, J. Quant. Spectros. Radiat. Transf., 110, 533

Sagawa, H., Hartogh, P., Rengel, M., de Lange, A., \& Cavalié, T. 2010, Planet. Space Sci., 58, 1692

Sekine, Y., Genda, H., Sugita, S., Kadono, T., \& Matsui, T. 2011, Nature Geosci., 4, 359

Strobel, D. F. 2010, Icarus, 208, 878

Teanby, N. A., Irwin, P. G. J., de Kok, R., et al. 2009a, Icarus, 202, 620

Teanby, N. A., Irwin, P. G. J., de Kok, R., \& Nixon, C. A. 2009b, Roy. Soc. London Philos. Trans. Ser. A, 367, 697

Teanby, N. A., Irwin, P. G. J., Nixon, C. A., et al. 2012, Nature, 491, 732

Urban, J., Baron, P., Lauti, N., et al. 2004, J. Quant. Spectros. Radiat. Transf., 83,529

Vinatier, S., Bézard, B., Fouchet, T., et al. 2007, Icarus, 188, 120

Vinatier, S., Bézard, B., Nixon, C. A., et al. 2010, Icarus, 205, 559

Wilson, E. H., \& Atreya, S. K. 2004, J. Geophys. Res. Planets, 109, 6002

Yung, Y. L., Allen, M., \& Pinto, J. P. 1984, ApJS, 55, 465 